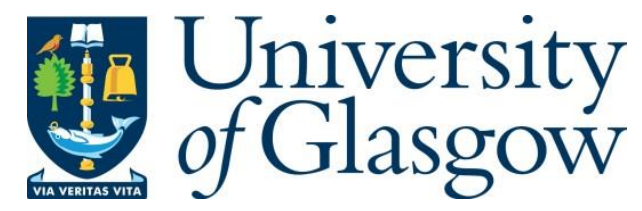

Correia-Melo, C., Ichim, G., Tait, S., and Passos, J. F. (2017) Depletion of mitochondria in mammalian cells through enforced mitophagy. Nature Protocols, 12(1), pp. 183-194. (doi:10.1038/nprot.2016.159)

This is the author's final accepted version.

There may be differences between this version and the published version. You are advised to consult the publisher's version if you wish to cite from it.

http://eprints.gla.ac.uk/140233/

Deposited on: 02 May 2017

Enlighten - Research publications by members of the University of Glasgow http://eprints.gla.ac.uk33640 


\section{Depletion of mitochondria in mammalian cells through enforced mitophagy}

Clara Correia-Melo ${ }^{1^{*}}$, Gabriel Ichim ${ }^{2,3^{*}}$, Stephen W.G. Tait ${ }^{2,3^{\wedge}}$, João F. Passos ${ }^{{ }^{\wedge}}$

${ }^{1}$ Institute for Cell and Molecular Biosciences, Campus for Ageing and Vitality, Newcastle University Institute for Ageing, Newcastle University, Newcastle upon Tyne, U.K. ${ }^{2}$ Cancer Research UK Beatson Institute, ${ }^{3}$ Institute of Cancer Sciences, University of Glasgow, Garscube Estate, Switchback Road, Glasgow, G61 1BD, U.K.

Subject Terms: Mitochondria, mitophagy, mitochondria-depleted cells, cell-death, senescence

* joint first authorship

$\wedge$ corresponding authors:

Dr. Stephen Tait, email: stephen.tait@glasgow.ac.uk Cancer Research UK Beatson Institute, Institute of Cancer Sciences, University of Glasgow, Garscube Estate, Switchback Road, Glasgow, G61 1BD, U.K.

Dr. João F. Passos, email: joao.passos@newcastle.ac.uk Newcastle University Institute for Ageing, Institute for Cell and Molecular Biosciences, Campus for Ageing and Vitality, Newcastle University, Newcastle upon Tyne, NE4 5PL, UK. Phone +44 191248 1222, Fax +441912481101, 


\section{Abstract}

Mitochondria are not only the "power-house" of the cell, but are also involved in a multitude of processes that include calcium storage, cell-cycle and cell-death. Traditional means to investigate mitochondrial importance in a given cellular process have centred upon depletion of mitochondrial DNA (mtDNA) through chemical or genetic means. While these methods severely disrupt mitochondrial electron transport chain, mtDNA-depleted cells still maintain mitochondria and many mitochondrial functions. Here, we describe a straightforward protocol to generate mammalian cell populations with low to non-detectable levels of mitochondria. Ectopic expression of the ubiquitin E3 ligase Parkin, combined with short-term mitochondrial uncoupler treatment, engages widespread mitophagy, and effectively eliminates mitochondria. In this protocol, we explain how to generate mitochondria-depleted cells and a variety of methods to confirm mitochondrial clearance. Furthermore, we describe culture conditions to maintain mitochondrial-depleted cells with minimal loss of viability for longitudinal studies. This method should prove useful to investigate the importance of mitochondria in a variety of biological processes.

\section{Introduction}

Mitochondria have traditionally been viewed as the powerhouse of the cell due to their central role in metabolism and energy production. More recently, mitochondria have been implicated in a wide-variety of other cellular roles including calcium storage, migration, cell death, senescence and inflammation ${ }^{1}$. Equally, impairment of mitochondrial homeostasis has been heavily linked to a wide range of diseases ${ }^{2,3}$; for this reason understanding the mechanisms that regulate mitochondrial health is of fundamental importance. To this end, eukaryotic cells are equipped with a range of mitochondrial quality control (QC) mechanisms 4,5 .

Mitochondria-specific autophagy, hereafter termed mitophagy, is a key mitochondrial QC mechanism that helps maintain mitochondrial fitness by efficiently removing dysfunctional organelles. While several different mechanisms regulating mitophagy have been described in mammalian cells, the best understood is PINK1/PARKIN-mediated mitophagy ${ }^{6}$. In this process, mitophagy occurs primarily following a complex interplay between the Ser/Thr kinase PINK1 and the E3 ubiquitin ligase PARKIN ${ }^{4}$. In healthy mitochondria PINK1 is subject to constant turnover; cleavage of its mitochondrial targeting sequence by the mitochondria intermembrane space protease PARL reveals a destabilizing $\mathrm{N}$-terminal residue that targets PINK for proteasomal degradation ${ }^{7,8}$. Disruption of mitochondrial function causes an accumulation of PINK1 on the mitochondrial outer 
membrane where it phosphorylates ubiquitin and Parkin leading to activation of the PARKIN E3 ubiquitin ligase at the mitochondria ${ }^{9-11}$. Parkin recruitment to mitochondria requires mitofusin 2 (MFN2) in some settings ${ }^{12,13}$ but is dispensible in others ${ }^{14}$. In a feed-forward mechanism, Parkin ubiquitinates mitochondrial substrates that, in turn, leads to more PINK1 substrate phosphorylation and Parkin activity. Ubiquitinated mitochondria are targeted for autophagy following recognition by specific ubiquitin binding adaptor proteins ${ }^{9}$. Importantly, experimentally induced PINK1/Parkin-mediated mitophagy can reduce mitochondrial levels to below the level of detection, thereby opening its use as an experimental tool to study mitochondrial function ${ }^{15,16}$.

\section{Development of an assay that allows the generation of mitochondria-depleted cells}

A well-established approach to study mitochondrial function in various processes centres upon depleting mitochondrial DNA (mtDNA) to generate so-called $\rho^{0}$ cells ${ }^{17}$. Typically this is achieved by long-term culture in the presence of ethidium bromide $(\mathrm{EtBr})$ that inhibits replication of mitochondrial but not nuclear DNA. Nevertheless, this method has various drawbacks including the extended time required to generate $\rho^{0}$ cells (typically weeks) and potential unwanted mutagenic effects on genomic DNA caused by $\mathrm{EtBr}$ treatment. Alternative methods to deplete mtDNA and generate $\rho^{0}$ cells include mitochondrial targeting of the restriction endonuclease EcoRI ${ }^{18}$ or expression of a dominant negative form of Poly, a gene that codes for the catalytic subunit of the mitochondrial DNA polymerase ${ }^{19}$. However, these solutions do not circumvent a major drawback, which is that $\rho^{0}$ cells still contain functional mitochondria. For example, mitochondrial apoptosis proceeds efficiently in $\rho^{0}$ cells 20. This prompted us to develop a method to remove mitochondria, allowing the study of cellular functions in the absence of all aspects of mitochondrial biology.

Herein, we describe a facile protocol that exploits PINK1/Parkin-mediated mitophagy as a tool to investigate mitochondrial function. In short, the method entails overexpression of Parkin and short-term mitochondrial depolarization to stimulate widespread mitophagy. This generates mammalian cells with low to non-detectable mitochondrial content allowing comparison of matched cells proficient or deficient in mitochondria (Figure 1). Demonstrating the protocol's effectiveness mitochondria-depleted cells show no detectable mitochondrial respiration, proteins or mtDNA, and strikingly no mitochondrial organelles were observed by 3D electron microscopy ${ }^{15,16}$. Following mitochondrial depletion, cells fail to repopulate their mitochondrial population over extended periods of time (16 days post removal of CCCP), demonstrating effective depletion. Furthermore, we have developed culture conditions to keep mitochondrial-depleted cells in culture with minimal loss of viability for long periods of time ranging from 5 days to 1 month (depending on the cell type) allowing extensive longitudinal studies. 


\section{Applications of the widespread-mitophagy protocol}

\section{Investigating the role of mitochondria in cell death}

Mitochondria are often essential in the execution of programmed cell death (or apoptosis). The intrinsic pathway of apoptosis is also called "mitochondrial apoptosis" since the permeabilisation of mitochondria is required for caspase activation and cell death ${ }^{21}$. A recently described non-apoptotic type of cell death is necroptosis (programmed necrosis) that requires the executioner protein mixed lineage kinase domain-like (MLKL) ${ }^{22}$. Mitochondria, through the generation of ROS, were considered essential for the execution of necroptosis ${ }^{23-25}$. To directly test this, we used Parkin-mediated mitophagy to efficiently remove mitochondria and assess its effect on necroptosis and apoptosis. As expected, mitochondrial-depleted cells were resistant to mitochondrial apoptosis ${ }^{15}$. However, while Parkin-mediated mitophagy completely prevented ROS production, it did not impact on necroptosis execution. This provided the first evidence that mitochondria are dispensable for the execution of necroptosis ${ }^{15}$.

\section{Investigating the role of mitochondria in cellular senescence}

Senescence is a cellular response to a variety of stresses, including oncogene activation, DNA damage and telomere dysfunction, that permanently arrests the cell. Senescence has been involved in several processes including development, cancer and tissue remodelling. Furthermore, increasing evidence suggests that senescence are major contributors to agerelated tissue dysfunction and pathologies, mostly by the development of a pro-oxidant and pro-inflammatory phenotype ${ }^{26}$. Mitochondrial dysfunction is a feature of senescence ${ }^{27}$ and has been shown to induce and stabilise the senescence arrest mostly via generation of ROS-driven cellular damage ${ }^{28}$. Despite the fact that multiple biochemical reactions occur within mitochondria, it is still relatively unknown what other factors apart from ROS impact on the senescent phenotype. By inducing Parkin-mediated widespread mitophagy our group has demonstrated that these organelles are required for the development of both the prooxidant and pro-inflammatory senescence-associated phenotypes ${ }^{16}$. This approach demonstrated the requirement of mitochondria for the development of several features of the senescent phenotype which are considered drivers of the ageing process, suggesting these organelles as putative therapeutic targets for interventions to inhibit senescence and ageing.

\section{Mitophagy as readout of autophagic flux}


Autophagy is a recycling process delivering various cytosolic cargoes (including whole organelles such as mitochondria or the peroxisomes) to lysosomes for degradation. Autophagy has lately received considerable attention due to its involvement in a multitude of

human diseases, ranging from neurodegenerative disorders to cancer ${ }^{29,30}$. It is therefore important to develop reliable methods for assessing the autophagic flux in vitro. This is especially relevant when testing new autophagy inhibitors that could be translated into clinical trials. The most commonly used method to characterize autophagy is the western blotting or immunofluorescence detection of lipidated LC3 (or LC3-II) which is indicative of autophagosome assembly. Nevertheless, inhibition of autophagosome breakdown or stimulation of autophagy can both increase LC3 II levels thereby complicating analysis. As such, a quantitative measure of autophagic flux is a much preferable measure. To address this issue, our lab has recently used the Parkin-mediated mitophagy protocol as a read-out of autophagic flux ${ }^{31}$. Briefly, mitophagy was used to detect differences in the autophagic flux between various cell lines with accurate detection and characterization of the effect of pharmacological or genetic inhibition/promotion of autophagy ${ }^{31}$.

\section{Additional Applications}

Generation of transmitochondrial cybrids. Using this method, mitochondria can be eliminated from cells followed by cell fusion with cytoplasts containing different degrees of mtDNA mutations. This method allows the investigation of mitochondrial diseases, without the need to use of $\rho^{0}$ cells- which still contain dysfunctional mitochondrial organelles.

Investigating mitochondrial biogenesis: We have found that if lower concentrations of CCCP are used and incomplete Parkin-mediated mitochondrial clearance occurs, mitochondria can repopulate. This will allow cell studies to investigate kinetics and determinants of mitochondrial replication.

Drug/genetic screens aiming to identify novel pathways that are dependent/independent of mitochondrial function.

\section{Advantages, limitations and adaptations}

Traditional methods to perturb mitochondrial function have relied on deletion of mtDNA, typically through long-term ethidium bromide treatment ${ }^{17}$. Nevertheless, while this effectively depletes mtDNA, mitochondria themselves and many of its associated functions remain in the cell. Moreover, it takes a considerable amount of time to achieve mtDNA depletion (weeks to months) and ethidium bromide may exert unwanted effects upon nuclear DNA. The protocol described below, using the Parkin-mediated mitochondrial depletion, addresses these issues by completely removing mitochondria in a quick (24 - 48 hours) and specific 
manner. Furthermore, this approach enables the generation of cell populations that show high penetrance of mitochondrial depletion (over $95 \%$ of cells with no detectable mitochondria) and therefore greatly facilitates the study of mitochondria in various cellular processes.

Potential limitations of this assay are that some cells do not readily survive mitochondrial depletion. In our own experience, all cell lines we have used show minimal cytotoxicity in reponse to short-term CCCP or antimycin A/oligomycin treatment. However, activation of Parkin-mediated mitophagy can cause extensive caspase-dependent apoptosis in some cell lines (HeLa for example). For unclear reasons, this cytoxicity appears cell-type dependent nevertheless it can be prevented by the co-application of caspase inhibitors. Secondly, the use of chemicals such as CCCP to induce mitophagy can have unwanted off-target effects ${ }^{32}$. Additionally, effective mitochondrial depletion requires ectopic expression of Parkin which may exert unwanted effects on the biological process being investigated. An approach to circumvent this may be through the use of an inducible Parkin expression vector, such that Parkin expression is switched off post-mitophagy. Finally, while the transduction of immortalised cells is often highly efficient, generation of stable cell lines using primary cells can be more troublesome - below we describe a protocol for both immortalised and primary cell lines.

\section{Experimental design}

The protocol can be divided into three main parts: generation of a stable YFP-Parkinexpressing cell line (Steps 1-10); induction of widespread mitophagy (Steps 11-13); and long-term culture of mitochondria-depleted cells. We finish with a brief summary for readouts of widespread mitophagy.

Generation of a stable YFP-Parkin-expressing cell line. In this protocol, we describe how to generate a stable YFP-Parkin-expressing cell line in primary human fibroblasts. Nevertheless, similar procedures have been successfully tested in other human primary (e.g., IMR-90 primary) and immortalised cells (e.g. KP-4 and HeLa cells) and murine cells (e.g. SVEC and $3 T 3$ cell). We also describe cell culture conditions were primary fibroblasts can be maintained viable for up to a month following mitochondrial depletion allowing for kinetics assays and long-term studies.

In this protocol we use Phoenix Amphotropic cells to produce retroviral particles. Phoenix ${ }^{\text {TM }}$ Amphotropic packaging cell lines are second generation retrovirus producer lines that were generated from human embryonic kidney (HEK) 293T cells ${ }^{33,34}$. In addition to the temperature sensitive $T$ antigen co-selected with neomycin already present in HEK293T 
cells, Phoenix ${ }^{\mathrm{TM}}$ Amphotropic cells contain two extra constructs: a construct capable of producing the gag-pol and a construct for the codification of the envelope protein for amphotropic viruses. Both the gag-pol and the envelope protein sequences were introduced with hygromycin and diphtheria toxin resistance as the co-selectable markers, respectively. The expression of each constructs is under regulation of different non-Moloney promoters to minimize recombination potential. The incorporation of these constructs in the Phoenix cells makes the transfection process relatively easy in these cells, where packaging cells (Phoenix ${ }^{\mathrm{TM}}$ Amphotropic cells), retroviral vectors and the transfection reagent are the only required transfection components (no requirement of additional packaging constructs). This cell line is highly transfectable with either calcium phosphate mediated transfection or lipidbased transfection protocols, with $\geq 50 \%$ cells being transiently transfected. In this protocol we use and describe a lipid-based transfection protocol using Lipofectamine ${ }^{\mathrm{TM}}$.

Next, we describe a protocol for transduction of YFP-Parkin retroviral particles into human primary fibroblasts. Human diploid fibroblasts, such as MRC5 fibroblasts, undergo a finite number of cell divisions or population doublings (PD) before permanently arresting in the cell cycle in a state termed replicative senescence ${ }^{35}$. Therefore, following a precise protocol when culturing and generating stable primary human fibroblasts cell lines is essential. For this reason, calculating the PD of human primary fibroblasts is required to evaluate the numbers of cell divisions that these cells have undergone and avoid transducing cells that are reaching their proliferative limit. Human fibroblasts are also sensitive to cell confluency: under confluency ( $\leq 40 \%$ cell density) or over confluency ( $\geq 100 \%$ cell density) can induce cellular stress and lead to premature senescence. It is thus necessary to calculate cell density for optimised cell fitness, transduction and posterior selection with antibiotics (e.g. Zeocin $^{\mathrm{TM}}$ ).

Induction of widespread Mitophagy. In this protocol, widespread mitophagy is achieved by inducing overall mitochondrial depolarisation. Carbonyl cyanide 3-chlorophenylhydrazone (CCCP) is a protonophore $\left(\mathrm{H}^{+}\right.$ionophore) and uncoupler of oxidative phosphorylation in mitochondria that promotes loss $\Delta \psi m$ and morphological swelling ${ }^{36}$. Treatment of YFPParkin-expressing cells with CCCP ensues mitochondrial dysfunction and translocation of Parkin to mitochondria, where it binds to PINK1 that has accumulated in the outermitochondrial membrane. An alternative to CCCP, as it can show off-target effects on lysosomal function ${ }^{32}$, is a mix of Antimycin A and Oligomycin A (in equimolar amounts) which are specific mitochondrial uncouplers. Typically, the widespread mitophagy protocol takes 48 hours. While immortalized cells require treatment with the mitochondrial membrane uncoupler every 12 hours, primary cells only require treatment every 24 hours for 48 hours. 
Note, the mitochondrial uncoupler dose and treatment duration should be tested for each cell line in order to efficiently induce widespread mitochondrial depletion.

\section{Long-term culture of mitochondria-depleted cells}

Here we describe the culture conditions that allow long-term culturing of cells depleted of mitochondria. This is mainly achieved by supplementing the growth media with uridine.

Assessment of mitophagy. The effectiveness of induced mitophagy can be assessed in multiple ways but typically relies upon detection of loss of mitochondrial protein(s) signal or loss of mtDNA. Importantly, Parkin has been shown to target some mitochondrial proteins for

proteasomal degradation independently of mitophagy ${ }^{37,38}$. Therefore, multiple approaches should be used, at least in initial experiments, to verify the method is functional.

\section{Materials}

\section{REAGENTS}

\section{Cell culture}

Critical: The cell lines used should be regularly checked to ensure that they are authentic and not infected with Mycoplasma. Although mycoplasma contamination does not affect induction of mitophagy it can affect several cellular functions and viability.

The generation of a YFP-Parkin expressing cell line involves the culturing of a packaging cell line for YFP-Parkin retroviral production, in this case Phoenix ${ }^{\mathrm{TM}}$ Amphotropic cells, and a cell line that will be subject to YFP-Parkin-retroviral transduction and become the YFP-Parkin expressing cell line (Table 1).

The indicated reagents or suppliers listed below can be substituted with appropriate alternatives if necessary.

- Dulbecco's modified Eagle's medium (DMEM, Cat. Number D5796, Sigma)

- Penicillin-Streptomycin (Cat. Number P4333, Sigma)

- Fetal bovine Serum (FBS, Cat. Number F9665, Sigma, Dorset)

- L-Glutamine (Cat. Number G7513, Sigma, Dorset)

- Trypsin-EDTA (TE) (Cat.Number T3924, Sigma) 
- $\quad$ Opti-MEM ${ }^{\circledR}$ I Medium (Cat. Number 11058-021, Thermo Fisher Scientic)

- MEM NEAA (Minimum Essential Medium Non-Essential Amino Acids) (Cat. Number 11140, Life Technologies)

- Sodium pyruvate (Cat. Number S8636, Sigma-Aldrich)

- 2-Mercaptoethanol (Cat. Number 21985, Life Technologies)

- Phosphate Buffer Saline (Cat. Number D1408, Sigma)

YFP-Parkin vector transfection and retroviral transduction

- YFP-Parkin-IRES-zeocin (Addgene, ID number 61728) Note: mCherry-Parkin-IRES-zeocin is also available (Addgene, ID number 61727)

- Lipofectamine ${ }^{\mathrm{TM}} 2000$ (Cat. Number 11668-019, Invitrogen,)

- Polybrene (Cat . Number sc-134220, Santa Cruz)

Caution: Polybrene is toxic if swallowed or in contact with the skin/eyes. Avoid contact with skin and eyes. Avoid formation of aerosols and provide appropriate exhaust ventilation at places where aerosols are formed.

Selection of efficiently transduced cells

- Zeocin $^{\mathrm{TM}}$ (Cat. Number R25001, Invitrogen)

Caution: Zeocin ${ }^{\mathrm{TM}}$ is toxic if swallowed or in contact with the skin/eyes. Zeocin ${ }^{\mathrm{TM}}$ is also suspected of causing genetic defects (mutagen). Avoid direct contact, and use gloves while preparing and using Zeocin ${ }^{\mathrm{TM}}$. Avoid formation of aerosols and provide appropriate exhaust ventilation.

Induction of mitophagy

- Carbonyl cyanide 3-chlorophenylhydrazone (CCCP, Cat number C2759, Sigma)

Caution: CCCP is toxic if swallowed, in contact with the skin/eyes or if inhaled. Avoid direct contact, and use gloves while preparing and using CCCP. Avoid formation of dust and aerosols. Provide appropriate exhaust ventilation.

- Antimycin A (Cat number A8674, Sigma)

Caution: Antimycin A is fatal if swallowed and toxic if in contact with the skin/eyes or if inhaled. Avoid direct contact, and use gloves while preparing and using Antimycin A. Avoid formation of dust and aerosols. Provide appropriate exhaust ventilation at 
places where dust/ aerosols is formed. Antimycin A is also toxic to aquatic life and therefore requires disposal of contents/ container to an approved waste disposal plant.

- Oligomycin A (Cat number 75351, Sigma)

Caution: Oligomycin A is harmful if swallowed, in contact with the skin/eyes or if inhaled. Avoid direct contact, and use gloves while preparing and using Oligomycin A. Avoid formation of dust and aerosols. Provide appropriate exhaust ventilation at places where dust/aerosols is formed.

\section{Assessment of widespread mitophagy}

Detection of mitochondrial proteins

Detection of mitochondrial proteins using western blot and/or immunofluorescence staining can be performed using conventional protocols and reagents. In Table 2 we describe the antibodies used for mitochondrial proteins detection used in this protocol.

Detection of mitochondrial proteins can also be achieved by live-cell imaging of YFP-Parkin cells expressing a mitochondrial targeted protein (for instance mito-dsRRed; Clontech, Cat. Number 632421).

\section{Quantification of mtDNA abundance}

Detection of mtDNA is performed by qPCR. Below we describe the mtDNA and nuclear (control) primers for mtDNA analysis used in this protocol (Table 3).

\section{EQUIPMENT}

- Centrifuge tubes, $15 \mathrm{ml}$ (Cat. Number E1415-0200, Starlab)

- Centrifuge tubes, $50 \mathrm{ml}$ (Cat. Number E1450-0200, Starlab)

- Microcentrifuge tubes, $1.5 \mathrm{ml}$ (Cat. Number E1415-1510, Starlab)

- Refrigerators, 4 and $-20^{\circ} \mathrm{C}$

- Heracell ${ }^{\mathrm{TM}}$ 150i CO incubator (ThermoScientific)

- Microcentrifuge 5415R (Eppendorf) 
- Corning® cell culture flasks surface area $75 \mathrm{~cm} 2$, canted neck (Cat. Number CLS430641-100EA, Sigma)

- Corning® Costar® cell culture plates 6 well (Cat. Nu,mber CLS3506-100EA, Sigma)

- Corning® Costar® cell culture plates 12 well (Cat. Nu,mber CLS3512-100EA, Sigma)

- Corning Tissue Culture Treated Culture Dishes 100mm X 20mm (Cat. Nubmber CLS430167-500EA, Sigma)

- Cover Glass, $\varnothing 19 \mathrm{~mm}$, Thickness 0.16mm (Cat. Number 631-0156, VWR)

- Millex-HV $0.45 \mu \mathrm{m}$ PVDF filters (Millipore, cat. no. SLHVR25LS)

- 35 mm glass-bottom dishes (Cat Number P35G-1.5-20-C, MatTek)

- Nikon TE2000 microscope with Perfect Focus System (PFS). The system is equipped with a Sutter Lambda 10-3 controller, ASI MS2 XY stage with linear encoders, Photometrics CoolSnap HQ CCD camera and Metamorph 7.5 software.

\section{REAGENT SETUP}

Culture medium for human Phoenix Amphotropic cells

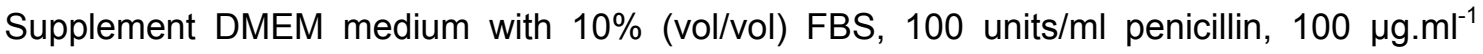
streptomycin, $2 \mathrm{mM}$ glutamine, $1 \mathrm{mM}$ sodium pyruvate, $300 \mu \mathrm{g} \cdot \mathrm{ml}^{-1}$ Hygromycin B and 1 $\mu \mathrm{g} \cdot \mathrm{ml}^{-1}$ Diphtheria toxin. This medium can be stored at $4{ }^{\circ} \mathrm{C}$ for up to 1 month.

\section{Culture medium for human MRC5 fibroblasts}

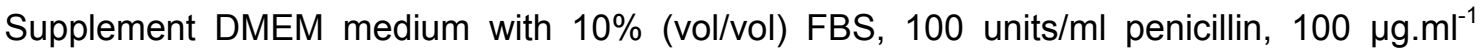
streptomycin and $2 \mathrm{mM}$ glutamine. This medium can be stored at $4{ }^{\circ} \mathrm{C}$ for up to 1 month.

\section{Culture medium for human MRC5 fibroblasts without mitochondria}

Supplement DMEM medium with 10\% (vol/vol) FBS, 100 units $/ \mathrm{ml}$ penicillin, $100 \mu \mathrm{g} \cdot \mathrm{ml}^{-1}$ streptomycin, $2 \mathrm{mM}$ glutamine and $50 \mu \mathrm{g} \cdot \mathrm{ml}^{-1}$ uridine. This medium can be stored at $4{ }^{\circ} \mathrm{C}$ for up to 1 month.

\section{Mitochondrial membrane uncouplers}

- Dissolve CCCP in DMSO to a stock concentration of $125 \mathrm{mM}$. Dilute to a final concentration of $12.5 \mu \mathrm{M}(1: 10000)$ in complete medium, just before adding it to the 
cells (add the same volume of DMSO to complete medium if needed a vehicle control). CCCP (stock solution of $125 \mathrm{mM}$ ) can be stored at $-20{ }^{\circ} \mathrm{C}$, protected from light for 6 months.

Critical: Prepare small aliquots of CCCP stock solution $(125 \mathrm{mM})$ and freeze them at $-20^{\circ} \mathrm{C}$. Avoid freeze/thawing cycles as this may reduce CCCP efficiency.

- Dilute Antimycin A and Oligomycin A in ethanol (100\%) to a stock concentration of 1 $\mathrm{mM}$ and small volume aliquots $(20-50 \mu \mathrm{l})$ are kept at $-20^{\circ} \mathrm{C}$. Dilute to a final concentration of $1 \mu \mathrm{M}(1: 1000)$ in complete medium, just before adding to the cells (add the same volume of ethanol to complete medium if needed a vehicle control). Antimycin A and Oligomycin A solutions can be stored at $-20^{\circ} \mathrm{C}$ for 6 months.

\section{Procedure}

\section{Generation of YFP-Parkin-expressing cell line}

Steps 1 to 4 summarize the transfection of YFP-Parkin vector into Phoenix ${ }^{\mathrm{TM}}$ Amphotrophic cell and generation of retroviral particles. Timing: 4-5 days

Critical step: Transfection protocols are performed following Biosafety level 2 (BL-2) procedures.

\section{Day 1}

Seed $5 \times 10^{6}$ Phoenix ${ }^{\mathrm{TM}}$ Amphotropic cells in $10 \mathrm{ml}$ of growth medium containing serum (no antibiotics) in a $10 \mathrm{~cm}$ tissue culture plate so that they will be $90-95 \%$ confluent on the next day (day of transfection).

2. Day 2

Before transfection, remove the culture medium from the Phoenix ${ }^{\mathrm{TM}}$ Amphotropic cells and replace with $5 \mathrm{ml}$ of $\mathrm{Opti}_{\mathrm{MEM}}{ }^{\circledR}$ । Medium containing serum (no antibiotics). For each transfection sample (pLZRS-YFP-Parkin and pLZRS-empty vector (EV) expression plasmid DNA), prepare DNA-Lipofectamine ${ }^{T M} 2000$ complexes as follows:

$2.1 \mathrm{In}$ sterile $15 \mathrm{ml}$ tubes, dilute $3 \mu \mathrm{g}$ of each expression plasmid DNA 
(pLZRS-EV and pLZRS-YFP-Parkin) in $1.5 \mathrm{ml}$ of Opti-MEM ${ }^{\circledR}$ । Medium without serum and mix gently.

$2.2 \mathrm{In}$ a separate sterile $15 \mathrm{ml}$ tube, dilute $9 \mu$ of Lipofectamine ${ }^{\mathrm{TM}} 2000$ in $1.5 \mathrm{ml}$ of Opti-MEM ${ }^{\circledR}$ । Medium without serum. Mix gently and incubate for 5 minutes at room temperature $\left(20^{\circ} \mathrm{C}\right)$. Note: Mix Lipofectamine ${ }^{\mathrm{TM}}$ 2000 gently before use.

2.3 After a 5-minute incubation, combine the diluted DNA with the diluted Lipofectamine $^{T M} 2000$ and mix gently. Allow for DNALipofectamine $^{T M} 2000$ complexes formation by incubating for 20 minutes at room temperature. The solution may appear cloudy, but this does not impede the transfection.

2.4 Add the DNA-Lipofectamine ${ }^{T M} 2000$ complexes dropwise to each plate of cells. Mix the plate gently by rocking back and forward and incubate cells overnight at $37^{\circ} \mathrm{C}$ in a humidified $5 \% \mathrm{CO}_{2}$ incubator.

Critical: Plasmid DNA for transfection into eukaryotic cells should very clean and free from contamination with phenol and sodium chloride. Contaminants can cause cytotoxicity, and salt will interfere with lipid complexing thereby decreasing transfection efficiency.

3. Day 3

Remove medium containing the DNA- Lipofectamine ${ }^{T M} 2000$ complexes and replace with $10 \mathrm{ml}$ complete culture medium (no antibiotics). Incubate at $37^{\circ} \mathrm{C}$ in a humidified $5 \%$ $\mathrm{CO}_{2}$ incubator.

4. Day $4-5$

4.1 Collect virus-containing supernatants 48 hours post-transfection into to a 15 $\mathrm{ml}$ sterile, capped, conical tube. Collection of virus-containing supernatants can also be done 72 hours post-transfection, however minimal differences in viral yield are observed whether supernatants are collected at either 48 or 72 hours posttransfection.

Caution: At this stage, follow the guidelines for working with BL-2 organisms. 
4.2 Centrifuge virus-containing supernatants at $1600 \mathrm{~g}$ for 15 minutes at $4^{\circ} \mathrm{C}$ to pellet debris, followed by supernatant filtration through a Millex-HV $0.45 \mu \mathrm{m}$ or equivalent PVDF filter. This step prevents contamination of packaging cells (retroviral producers) into the cell line to be transduced.

Caution: At this stage, follow the guidelines for working with BL-2 organisms.

Steps 5 to 10 are a brief protocol of YFP-Parkin retroviral transduction in human primary fibroblasts. The procedure is similar for other mammalian cell lines.

Timing: $15-17$ days

Critical: Transduction protocols are performed following BL-2 procedures.

Critical: When using human primary cells, use cells at a lower PD in order to avoid producing a cell line that by the end of the transduction process is senescent or approaching senescence. To calculate the PD, cells should be trypsinized and counted for total number of cells calculation. The following formula should then be applied: $P D=X \pm(\operatorname{Ln}(n 2 / n 1) / L n 2$, where $X$ is the number of the accumulated PD; $n 2$ is the total number of cells obtained and $\mathrm{n} 1$ is the number of cells initially seeded in the plate.

\section{Day 1}

Seed $2 \times 10^{5}$ cells/well into a 6 -well plate (cells should be $50-60 \%$ confluent at the time of transduction). Incubate cells at $37^{\circ} \mathrm{C}$ overnight in a humidified $5 \% \mathrm{CO}_{2}$ incubator.

Critical: Primary human fibroblasts do not form colonies when proliferating and therefore do not allow for viral titering, which permits optimization of viral transduction volumes for efficient transductions. Furthermore, efficiency of transfection and viral particles generation can vary from experiment to experiment and with the size of the plasmid DNA. We advise performing transduction with several viral-supernatant volumes. Example: seed 3 wells in a 6-well plate: 1) for $1 \mathrm{~mL}$ viral transduction; 2) for 2 $\mathrm{mL}$ viral transduction and 3 ) for non-transduced control (mock).

\section{Day 2}

Add the purified media with $10 \mu \mathrm{g} / \mathrm{ml}$ polybrene to cells at $50-60 \%$ cell confluency. Incubate at $37^{\circ} \mathrm{C}$ overnight in a humidified $5 \% \mathrm{CO}_{2}$ incubator. Note that this step should be done immediately after step 4.2, thus seeding the cells for transduction (step 5) 
should be performed the day before to viral collection. Alternatively, viral particles can be frozen at $-80^{\circ} \mathrm{C}$ after collection and purification (note that in some cases transduction efficiency can be affected if using viral particles that were previously frozen; avoid freeze/thaw cycles).

\section{Day 3}

Remove the media containing viral particles and refresh the media with $2 \mathrm{ml}$ of complete culture medium. Incubate at $37^{\circ} \mathrm{C}$ overnight in a humidified $5 \% \mathrm{CO}_{2}$ incubator.

Caution: Viral particle containing medium should be disposed according to BL-2 safety procedures.

8. Day 4

Remove the medium and wash the cells once with PBS. Trypsinize the cells and replate all cells (eachwell of a 6-well plate) into one $10 \mathrm{~cm}$ plate containing complete culture medium. Incubate at $37^{\circ} \mathrm{C}$ overnight in a humidified $5 \% \mathrm{CO}_{2}$ incubator.

Caution: Medium containing residual viral particles should be disposed according to BL2 safety procedures.

9. Day 5

For Zeocin ${ }^{\mathrm{TM}}$ selection, add $10 \mathrm{~mL}$ complete culture medium with $250 \mu \mathrm{M}$ Zeocin ${ }^{\mathrm{TM}}$ to select for stably transduced cells. Human primary fibroblasts should be at $40-50 \%$ cell confluency when adding Zeocin ${ }^{\mathrm{TM}}$ for maximum selection efficiency. Replace medium with fresh medium containing $250 \mu \mathrm{M}$ Zeocin ${ }^{\mathrm{TM}}$ every 3-4 days.

Note: If using a different cell line, prior to this step a survival curve with different Zeocin $^{\mathrm{TM}}$ concentrations should be performed on non-transduced cells to select for the lowest dose of Zeocin ${ }^{\mathrm{TM}}$ that causes $100 \%$ cell death.

10. Day $15-17$

After 10-12 days of selection (day 14-16), stably YFP-Parkin expressing fibroblasts should have repopulated the transduced dish and no live cells should be observed in the 
mock dish. Remove the medium and replace with complete media. During this period, efficiently transduced cells may need to be passaged into bigger flasks as they reach confluency.

Critical: Store Zeocin ${ }^{\mathrm{TM}}$ at $-20^{\circ} \mathrm{C}$ and thaw on ice before use. Zeocin ${ }^{\mathrm{TM}}$ is light sensitive. Store the drug, and medium containing drug, in the dark at $4^{\circ} \mathrm{C}$. Culture medium containing Zeocin ${ }^{T M}$ may be stored at $4^{\circ} \mathrm{C}$ protected from exposure to light for up to 1 month.

\section{Induction of widespread mitophagy}

Timing: 4 days, up to 6 days

In this protocol, we describe general conditions for the induction of widespread mitophagy in primary cells. Despite following a similar protocol, primary and immortalised cells can differ in a few aspects of the protocol; below we describe the details regarding these differences.

Critical: Cell density prior and during induction of widespread mitophagy can impact on the success of the protocol.

11. Day 1

11.1 Seed $1.5 \times 10^{6}$ human primary fibroblasts (MRC5 or IMR90) $(20<P D<25)$ into 10 $\mathrm{cm}$ culture dishes in $10 \mathrm{ml}$ of complete culture medium. Incubate cells at $37^{\circ} \mathrm{C}$ overnight in a humidified $5 \% \mathrm{CO}_{2}$ incubator.

11.2 Seed cells for mitochondria depletion QC assays: $5 \times 10^{5}$ cells/well in 6-well plates, for mitochondrial protein expression analysis by western blot or mtDNA copy number by $\mathrm{qPCR}$, or $4 \times 10^{4}$ cells/well in coverslips in 12-well plates, for mitochondrial protein expression analysis by immunofluorescence staining, and incubate at $37^{\circ} \mathrm{C}$ overnight in a humidified $5 \% \mathrm{CO}_{2}$ incubator.

Note: Mitochondria depletion QC assays should be performed immediately after removal of the mitophagy inducer drug. Nevertheless, if the main experiment requires culturing cells for longer (e.g. 2 days after removal of the mitochondria uncoupler drug), then extra cells should be seeded and harvested, at the same time as the cells in the main experiment, for QC assays.

Critical: Cells should be $70 \%$ confluent the next day. The number of cells seeded is dependent on the PD value, with advancing PDs cells start to become bigger (presenescent), which impacts on cell culture density (e.g. lower PD cells require to be 
seeded in higher density). It is, therefore, critical to adjust the number of cells to the PD value.

Note: Other cell lines, particularly immortalised cells, may require different cell culture density when applying this protocol (e.g. HeLa cells should be at $10-20 \%$ confluency prior induction of mitophagy for optimal efficiency of the protocol).

12. Day $2-3$

Treat cells every 24 hours, during 48 hours, with $12.5 \mu \mathrm{M}$ CCCP in complete medium. Note: Unlike primary cells, Immortalised cells (e.g. KP-4, HeLa, 3T3 and SVEC cells) are treated every 12 hours, during 48 hours, with $12.5 \mu \mathrm{M} \mathrm{CCCP} \mathrm{in} \mathrm{complete} \mathrm{medium.}$ Cells can also be treated with $1 \mathrm{uM}$ antimycin $\mathrm{A}$ and $1 \mathrm{uM}$ oligomycin $\mathrm{A}$ in complete culture medium. If using other cell lines that are not mentioned in this protocol, the mitochondrial uncoupler dose and treatment duration may need to be adjusted.

Critical: $\mathrm{CCCP}$, antimycin A and oligomycin A are not stable in the media for longer periods of time and therefore need to be refreshed at least every 24 hours.

13. Day 4

Most cells lines tested have undergone extensive mitophagy at this point. At this stage, harvest cells for mitochondria depletion QC assays and proceed with the main experiment.

13.1 Cells for mitochondrial depletion QC assays. Remove the medium containing CCCP and rinse cells once in pre-warmed PBS. Harvest cells for mitochondria depletion QC assays according to a pre-determined method (see below).

13.2 Cells to proceed with the experiment. Remove the medium containing CCCP and rinse cells once in pre-warmed PBS. Refresh the medium with complete medium and incubate cells at $37^{\circ} \mathrm{C}$ in a humidified $5 \% \mathrm{CO}_{2}$ incubator, for the duration of the experiment (up to 2 days post mitochondria depletion).

\section{Long-term culture of mitochondria-depleted cells}

Timing: 4 days, up to 30 days 
Absence of mitochondria will impact on the function of the mitochondrial enzyme dihydroorotate dehydrogenase (DHODH) which is involved in pyrimidine synthesis (King and Attardi 1996). One way to overcome this is through the addition of uridine to the cell culture media. We have found that primary fibroblasts positively respond to the addition of uridine to the cell culture medium, allowing viability in culture for longer periods of time. Below, we describe how to culture mitochondria-depleted primary cells for a long period (up to 15 days in proliferating cells and up to 30 days in permanently arrested (senescent) cells).

\section{Day 1}

Seed $1.5 \times 10^{6}$ human primary fibroblasts $(20<P D<25)$ into a $10 \mathrm{~cm}$ dish in complete media and allow cells to adhere at $37^{\circ} \mathrm{C}$ overnight in a humidified $5 \% \mathrm{CO}_{2}$ incubator. Cells should be $70-90 \%$ confluent the next day. At the same time, seed cells for mitochondria depletion QC assays as described previously.

Critical: It is critical that cells are 70 to $90 \%$ confluent prior treatment with the mitochondria uncoupler. Cell density will depend on the duration of the experiment: the longer the experiment the higher the cell density required prior treatment, as mitochondria-depleted cells tend to become smaller with time, compromising optimal cell density/contact and viability.

15. Day 2 - 3

Treat cells every 24 hours, over a 48 hour period, with $12.5 \mu \mathrm{M} \mathrm{CCCP} \mathrm{in} \mathrm{complete}$ medium. Cells can also be treated with $1 \mathrm{uM}$ antimycin $\mathrm{A}$ and $1 \mathrm{uM}$ oligomycin $\mathrm{A}$ in complete culture medium.

Critical: $\mathrm{CCCP}$, antimycin A and oligomycin A are not stable in the media on cells for longer periods of time and therefore need to be refreshed at least every 24 hours. Different primary cells may need adjusting drug refreshment periods.

16. Day 4

Remove medium containing CCCP and replace with complete medium with additional $50 \mathrm{ug} / \mathrm{ml}$ uridine. Incubate cells at $37^{\circ} \mathrm{C}$ in a humidified $5 \% \mathrm{CO}_{2}$ incubator. At this point cells should de devoid of mitochondria, collect cells for mitochondria 
depletion QC assays, as described previously.

\section{Day 5-up to 15}

Refresh medium supplemented with $50 \mathrm{ug} / \mathrm{ml}$ uridine every 2 days until the end of your experiment. By the end of the experiment harvest cells for the main experiments and cells for mitochondria depletion QC assays as described previously.

Critical: In long-term experiments, it is critical for cell fitness and viability that cells are cultured in medium supplemented with uridine, as it will partially overcome the limiting pyrimidine levels in mitochondria-depleted cells. Secondly, mitochondrial depletion should also be assessed at long-term post-depletion, to ensure viable cells are not simply the ones that escaped initial depletion.

\section{Readouts of widespread mitophagy}

We suggest the following conventional methods for determination of widespread mitochondria: western blotting (see Figure $2 A \& G$ ), immunofluorescence staining for specific mitochondrial proteins followed by microscopy and/or flow cytometry analysis (see Figure $2 \mathrm{D}$ $\& E$ ) and $q P C R$ for mtDNA copy number (see Figure 2E). Antibodies used for Western blotting and Immmunofluorescence and primers for mtDNA copy number assays are described in the Material section.

Furthermore, we can detect clearance of mitochondria by live cell imaging of YFP-Parkin cells expressing a mitochondrial targeted protein (for instance mito-dsRRed) (See video Figure 1). Briefly, for live-cell imaging, plate YFP-Parkin expressing cells, transiently expressing mitochondrial targeted dsReD (mito-dsRed), on $35 \mathrm{~mm}$ glass-bottom dishes and image using a long-term time lapse Nikon TE2000 microscope. Prior to imaging, treat cells with antimycin and oligomycin using the protocol described above.

\section{Troubleshooting}

Troubleshooting advice can be found in Table 4.

\section{Timing}

Generation of Parkin encoding retrovirus, $4-5$ days. Retroviral transduction and selection of Parkin expressing cells, $15-17$ days. Induction of widespread mitophagy, $4-5$ days. Long 
term culture of mitochondria-depleted cells, up to 30 days. QC analysis of mitochondrial depletion, $2-3$ days.

\section{Anticipated results}

In Figure 2, we show different experiments that validate the complete removal of mitochondria using the widespread mitophagy protocol. In panel A, KP-4 YFP-Parkin cells were treated with $\mathrm{A} / \mathrm{O}$ for 48 hours and then immunoblotted with Mitoprofile. In panels $\mathrm{B}$ and $C$ the same cells were immunostained for COXIV and TIMM44 and no mitochondria were observed. In panel D, we confirm mitochondrial depletion by using FACS for the same proteins as in $\mathrm{B}$ and $\mathrm{C}$. In panel $\mathrm{F}$ we illustrate the procedure for clearance of mitochondria followed by long-term culture of cells (only applicable to human primary fibroblasts). In panel $G$ we show expected western blotting results for several mitochondrial proteins: NDUFB8, SDHA, UQCRC2 and TOMM20 at day 4 and 16 after removal of CCCP. These results demonstrate that the initial CCCP treatment is sufficient for clearance of mitochondria and no repopulation occurs at later stages.

\section{Figure legends}

\section{Figure 1. Overview of enforced mitophagy protocol}

Cells stably expressing YFP-Parkin are treated with CCCP or a combination of Antimycin A and Oligomycin A for up to $48 \mathrm{~h}$. This disrupts the mitochondrial inner membrane potential $(\Delta \psi \mathrm{m})$, leading to PINK1 accumulation on the outer mitochondrial membrane and Parkin activation and recruitment at the mitochondria. Here, Parkin ubiquitinates a multitude of mitochondrial proteins that serves as signal for autophagosome engulfment of damaged mitochondria. Ultimately, all mitochondria are removed via mitophagy.

\section{Figure 2. Validating Parkin-mediated widespread mitophagy}

A. KP-4 cells stably expressing YFP-Parkin were treated with antimycin A and oligomycin A (AO) for 48 hours and then immune-blotted with a cocktail of antibodies recognizing various mitochondrial proteins. B-C. Following induction of mitophagy, KP-4 YFP-Parkin cells were immuno-stained for the mitochondrial proteins COXIV (B) and TIMM44 (C). Scale bar is 50 $\mu \mathrm{m}$. D. Flow cytometry analysis showing reduced levels of COXIV and TIMM44 in AO-treated KP-4 cells expressing YFP-Parkin. E. mtDNA quantitative PCR on KP-4 YFP-Parkin cells after induction of mitophagy. A representative experiment (out of two) is shown with error bars representing standard deviation for three technical replicates. F. Workflow of widespread 
mitophagy and long-term culture of human primary fibroblast with depleted mitochondria.

G. Western blot analysis for mitochondrial content in primary fibroblasts 4 and 16 days after CCCP release.

\section{Author contribution statements}

C.C-M.,G.I., S.T. and J.P. developed the protocol. C.C-M.,G.I., S.T. and J.P designed the experiments described herein. G.I. and C.C-M. performed the experiments, interpreted the results and prepared figures. C.C-M.,G.I., S.T. and J.P. wrote the manuscript.

\section{Acknowledgments}

We thank Jaclyn Long and Kevin Ryan for providing KP-4 cells expressing Parkin. CCM was funded by Newcastle University and Foundation for Science and Technology through the GABBA Programme, University of Porto. G.I. was supported by an EMBO advanced longterm postdoctoral fellowship (ALTF 55-2013). This research was supported by the BBSRC (grant BB/K008374/1) and the Royal Society (both S.T). S.T is a Royal Society University Research Fellow. Work in the JFP lab was funded by a David Phillips Fellowship BB/H022384/1 and a BBSRC grant BB/K017314/1. We thank Catherine Winchester (Beaston Institute) for reviewing the manuscript.

\section{Competing financial interests}

The authors declare that they have no competing financial interests

\section{References}

1 Chandel, N. S. Mitochondria as signaling organelles. BMC biology 12, 34, doi:10.1186/1741-7007-12-34 (2014).

2 Pickrell, A. M. \& Youle, R. J. The roles of PINK1, parkin, and mitochondrial fidelity in Parkinson's disease. Neuron 85, 257-273, doi:10.1016/j.neuron.2014.12.007 (2015).

3 Dorn, G. W., 2nd, Vega, R. B. \& Kelly, D. P. Mitochondrial biogenesis and dynamics in the developing and diseased heart. Genes Dev 29, 1981-1991, doi:10.1101/gad.269894.115 (2015).

4 Youle, R. J. \& Narendra, D. P. Mechanisms of mitophagy. Nat Rev Mol Cell Biol 12, 9-14, doi:10.1038/nrm3028 (2011).

5 Quiros, P. M., Langer, T. \& Lopez-Otin, C. New roles for mitochondrial proteases in health, ageing and disease. Nat Rev Mol Cell Biol 16, 345-359, doi:10.1038/nrm3984 (2015). 
Riley, J. S. \& Tait, S. W. Mechanisms of mitophagy: putting the powerhouse into the doghouse. Biological chemistry, doi:10.1515/hsz-2016-0137 (2016).

7 Jin, S. M. et al. Mitochondrial membrane potential regulates PINK1 import and proteolytic destabilization by PARL. J Cell Biol 191, 933-942, doi:10.1083/jcb.201008084 (2010).

8 Yamano, K. \& Youle, R. J. PINK1 is degraded through the $\mathrm{N}$-end rule pathway. Autophagy 9, 1758-1769, doi:10.4161/auto.24633 (2013).

9 Kane, L. A. et al. PINK1 phosphorylates ubiquitin to activate Parkin E3 ubiquitin ligase activity. J Cell Biol 205, 143-153, doi:10.1083/jcb.201402104 (2014).

10 Koyano, F. et al. Ubiquitin is phosphorylated by PINK1 to activate parkin. Nature 510, 162-166, doi:10.1038/nature13392 (2014).

11 Kazlauskaite, A. et al. Parkin is activated by PINK1-dependent phosphorylation of ubiquitin at Ser65. Biochem J 460, 127-139, doi:10.1042/BJ20140334 (2014).

12 Chen, Y. \& Dorn, G. W., 2nd. PINK1-phosphorylated mitofusin 2 is a Parkin receptor for culling damaged mitochondria. Science 340, 471-475, doi:10.1126/science.1231031 (2013).

13 Gong, G. et al. Parkin-mediated mitophagy directs perinatal cardiac metabolic maturation in mice. Science 350, aad2459, doi:10.1126/science.aad2459 (2015).

14 Narendra, D., Tanaka, A., Suen, D.-F. \& Youle, R. J. Parkin is recruited selectively to impaired mitochondria and promotes their autophagy. The Journal of Cell Biology 183, 795-803, doi:10.1083/jcb.200809125 (2008).

15 Tait, S. W. et al. Widespread mitochondrial depletion via mitophagy does not compromise necroptosis. Cell reports 5, 878-885, doi:10.1016/j.celrep.2013.10.034 (2013).

16 Correia-Melo, C. et al. Mitochondria are required for pro-ageing features of the senescent phenotype. EMBO J 35, 724-742, doi:10.15252/embj.201592862 (2016).

17 King, M. P. \& Attardi, G. Isolation of human cell lines lacking mitochondrial DNA. Methods in enzymology 264, 304-313 (1996).

18 Kukat, A. et al. Generation of rho0 cells utilizing a mitochondrially targeted restriction endonuclease and comparative analyses. Nucleic Acids Res 36, e44, doi:10.1093/nar/gkn124 (2008).

19 Jazayeri, M. et al. Inducible expression of a dominant negative DNA polymerasegamma depletes mitochondrial DNA and produces a rho0 phenotype. J Biol Chem 278, 9823-9830 (2003).

20 Jacobson, M. D. et al. Bcl-2 blocks apoptosis in cells lacking mitochondrial DNA. Nature 361, 365-369, doi:10.1038/361365a0 (1993).

21 Tait, S. W. \& Green, D. R. Mitochondria and cell death: outer membrane permeabilization and beyond. Nat Rev Mol Cell Biol 11, 621-632, doi:10.1038/nrm2952 (2010).

22 Tait, S. W., Ichim, G. \& Green, D. R. Die another way--non-apoptotic mechanisms of cell death. J Cell Sci 127, 2135-2144, doi:10.1242/jcs.093575 (2014).

23 Kim, Y. S., Morgan, M. J., Choksi, S. \& Liu, Z. G. TNF-induced activation of the Nox1 NADPH oxidase and its role in the induction of necrotic cell death. Mol Cell 26, 675687, doi:10.1016/j.molcel.2007.04.021 (2007).

24 Vanlangenakker, N. et al. clAP1 and TAK1 protect cells from TNF-induced necrosis by preventing RIP1/RIP3-dependent reactive oxygen species production. Cell Death Differ 18, 656-665, doi:10.1038/cdd.2010.138 (2011).

25 Cho, Y. S. et al. Phosphorylation-driven assembly of the RIP1-RIP3 complex regulates programmed necrosis and virus-induced inflammation. Cell 137, 11121123, doi:10.1016/j.cell.2009.05.037 (2009).

26 van Deursen, J. M. The role of senescent cells in ageing. Nature 509, 439-446, doi:10.1038/nature13193 (2014).

27 Correia-Melo, C. \& Passos, J. F. Mitochondria: Are they causal players in cellular senescence? Biochim Biophys Acta 1847, 1373-1379, doi:10.1016/j.bbabio.2015.05.017 (2015). 
28 Passos, J. F. et al. Feedback between p21 and reactive oxygen production is necessary for cell senescence. Mol Syst Biol 6, 347 (2010).

29 Metcalf, D. J., Garcia-Arencibia, M., Hochfeld, W. E. \& Rubinsztein, D. C. Autophagy and misfolded proteins in neurodegeneration. Experimental neurology 238, 22-28, doi:10.1016/j.expneurol.2010.11.003 (2012).

30 Rosenfeldt, M. T. \& Ryan, K. M. The multiple roles of autophagy in cancer. Carcinogenesis 32, 955-963, doi:10.1093/carcin/bgr031 (2011).

31 Baudot, A. D., Haller, M., Mrschtik, M., Tait, S. W. \& Ryan, K. M. Using enhancedmitophagy to measure autophagic flux. Methods 75, 105-111, doi:10.1016/j.ymeth.2014.11.014 (2015).

32 Padman, B. S., Bach, M., Lucarelli, G., Prescott, M. \& Ramm, G. The protonophore CCCP interferes with lysosomal degradation of autophagic cargo in yeast and mammalian cells. Autophagy 9, 1862-1875, doi:10.4161/auto.26557 (2013).

33 Pear, W. S., Nolan, G. P., Scott, M. L. \& Baltimore, D. Production of high-titer helperfree retroviruses by transient transfection. Proc Natl Acad Sci U S A 90, 8392-8396 (1993).

34 Swift, S., Lorens, J., Achacoso, P. \& Nolan, G. P. Rapid production of retroviruses for efficient gene delivery to mammalian cells using 293T cell-based systems. Current protocols in immunology / edited by John E. Coligan ... [et al.] Chapter 10, Unit 10 17C, doi:10.1002/0471142735.im1017cs31 (2001).

35 Hayflick, L. \& Moorhead, P. S. The serial cultivation of human diploid cell strains. Exp Cell Res 25, 585-621 (1961).

36 Minamikawa, T., Williams, D. A., Bowser, D. N. \& Nagley, P. Mitochondrial permeability transition and swelling can occur reversibly without inducing cell death in intact human cells. Exp Cell Res 246, 26-37, doi:10.1006/excr.1998.4290 (1999).

37 Yoshii, S. R., Kishi, C., Ishihara, N. \& Mizushima, N. Parkin mediates proteasomedependent protein degradation and rupture of the outer mitochondrial membrane. $J$ Biol Chem 286, 19630-19640, doi:10.1074/jbc.M110.209338 (2011).

38 Chan, N. C. et al. Broad activation of the ubiquitin-proteasome system by Parkin is critical for mitophagy. Human molecular genetics 20, 1726-1737, doi:10.1093/hmg/ddr048 (2011). 
Table 1 | Cell lines for the generation of a stable YFP-Parkin-expressing cell line

\begin{tabular}{lllll}
\hline Cell line & Species & Source & Cell type & Application \\
\hline Phoenix & Human & ATCC (Cat. & Immortalised & Viral particles \\
Amphotropic & & Number $\underline{\text { CRL- }}$ & & generation
\end{tabular}

3213)

MRC5

Fibroblasts

IMR-90

Fibroblasts

$\mathrm{KP}-4$

U2OS

MCF-7

HeLa
Human

(1)

Human

Human

Human

Human

Human

Number HTB-22)

Human ATCC (Cat.
RIKEN

ATCC (Cat.

\author{
ECACC, Salisbury, \\ UK (Cat. Number \\ 85020204)
}

ATCC (Cat.

Number HTB-96)

Immortalised

Immortalised

\section{Immortalised Viral transduction and generation of a stable YFP-PARKIN- \\ expressing cell line}

Viral transduction and generation of a stable YFP-PARKIN-

expressing cell line

Viral transduction and generation of a stable YFP-PARKINexpressing cell line Number CCL-2)
Immortalised Viral transduction and generation of a stable YFP-PARKIN- 
expressing cell line

3T3-SA

Mouse ATCC (Cat.

Immortalised Viral transduction and

Number CCL-92)

generation of a stable

YFP-PARKIN-

expressing cell line

NIH-3T3

Mouse

ATCC (Cat.

Immortalised

Viral transduction and

Number CRL-

1658)

generation of a stable

YFP-PARKIN-

expressing cell line

SVEC

Mouse

ATCC (Cat.

Immortalised Viral transduction and

Number CRL-

generation of a stable

2167)

YFP-PARKIN-

expressing cell line 
Table 2 | Antibodies for widespread mitophagy QC assays

Primary Antibodies

\begin{tabular}{|c|c|c|c|c|c|}
\hline Antibody & Species & Host & $\begin{array}{l}\text { Reference/ } \\
\text { Manufacturer }\end{array}$ & $\begin{array}{c}\text { WB } \\
\text { (dilution) }\end{array}$ & $\begin{array}{c}\text { IMF } \\
\text { (dilution) }\end{array}$ \\
\hline MitoProfile & Human & Mouse & ab110414/ & 1:1000 & \\
\hline Membrane & Mouse & monoclonal & Abcam & & \\
\hline Integrity & Rat & & & & \\
\hline antibody & Cow & & & & \\
\hline \multicolumn{6}{|l|}{ cocktail } \\
\hline \multirow[t]{2}{*}{ TIMM44 } & Human & Rabbit & ab194829/ & ------------- & $1: 200$ \\
\hline & Mouse & monoclonal & Abcam & - & \\
\hline \multirow[t]{4}{*}{ COXIV } & Human & Mouse & 11967/ & ------------ & $1: 200$ \\
\hline & Mouse & monoclonal & Cell Signaling & - & \\
\hline & Rat & & & & \\
\hline & Monkey & & & & \\
\hline \multirow[t]{2}{*}{ SDHA } & Human & Rabbit & \#11998/ & 1:1000 & $1: 100$ \\
\hline & Mouse & monoclonal & Cell Signaling & & \\
\hline \multirow[t]{2}{*}{ NDUFB8 } & Human & Mouse & ab110242 - & 1:1000 & \\
\hline & Mouse & monoclonal & Abcam & & \\
\hline \multirow[t]{3}{*}{ UQCRC2 } & Human & Mouse & ab14745 - & 1:1000 & \\
\hline & & monoclonal & Abcam & & \\
\hline & Mouse & & & & \\
\hline \multirow[t]{3}{*}{ TOMM20 } & & Mouse & ab56783 - & 1:1000 & \\
\hline & & monoclonal & & & \\
\hline & Mice & & & & \\
\hline
\end{tabular}




\begin{tabular}{|c|c|c|c|c|}
\hline \multirow[t]{2}{*}{ GAPDH } & Human & Rabbit & \#5174/ & $1: 5000$ \\
\hline & Mouse & monoclonal & Cell sigalling & \\
\hline \multirow[t]{3}{*}{$\alpha$-tubulin } & Human & Mouse & T6074/ & $1: 5000$ \\
\hline & Mouse & monoclonal & Sigma-Aldrich & \\
\hline & rat & & & \\
\hline \multirow[t]{3}{*}{ actin } & Mammals & Mouse & $8691001 /$ & $1: 5000$ \\
\hline & Yeast & monoclonal & MP & \\
\hline & Fungi & & Biomedicals & \\
\hline
\end{tabular}

Secondary Antibodies

\begin{tabular}{|c|c|c|c|}
\hline Secondary antibody & $\begin{array}{l}\text { Reference/ } \\
\text { Manufacturer }\end{array}$ & $\begin{array}{c}\text { WB } \\
\text { (dilution) }\end{array}$ & $\begin{array}{c}\text { IMF } \\
\text { (dilution) }\end{array}$ \\
\hline $\begin{array}{l}\text { Anti-rabbit Fluorescein- } \\
\text { conjugated secondary } \\
\text { antibody AlexaFluor } 647\end{array}$ & $\begin{array}{c}\text { A21244/ } \\
\text { Molecular Probes }\end{array}$ & & 1:1000 \\
\hline $\begin{array}{c}\text { Goat anti-rabbit IgG -HRP } \\
\text { conjugated }\end{array}$ & $\begin{array}{c}\text { A0545/ } \\
\text { Sigma-Aldrich }\end{array}$ & 1:5000 & \\
\hline $\begin{array}{c}\text { Rabbit anti-mouse IgG -HRP } \\
\text { conjugated }\end{array}$ & $\begin{array}{c}\text { A2554/ } \\
\text { Sigma-Aldrich }\end{array}$ & 1:5000 & \\
\hline
\end{tabular}




\begin{tabular}{llll}
\hline Gene & \multicolumn{2}{c}{ Primer Forward } & Primer Reverse \\
\hline mtDNA & MTRNR2 & CGAAAGGACAAGAGAAATAAGG & \\
mEDNA & MTTL1 & & CTGTAAAGTTTTAAGTTTTATGCG \\
nuclear & 6-Globin & CAACTTCATCCACGTTCACC & GAAGAGCCAAGGACAGGTAC \\
\hline
\end{tabular}

\begin{tabular}{llll}
\hline \multicolumn{3}{c}{ Table 4 | Troubleshooting } \\
\hline Step & Problem & Possible reason & Possible solution \\
\hline
\end{tabular}

1-10 None or few Inefficient viral transfection cells expressing that can be a result of:

YFP-Parkin

a) Loss of viral packaging It is important that cells are plasmid in the Phoenix ${ }^{\mathrm{TM}}$ previously selected with the Amphotropic cells during respective selecting replication. antibiotics (Hygromycin B and Diphteria toxin).

b) Suboptimal cell density. If Phoenix $^{\mathrm{TM}}$ Amphotropic cells cell density at the time of should be $90-95 \%$ confluent transfection is suboptimal by the time of transfection.

(low cell density) cells may not be in the optimal growth phase for transfection. This can result in insufficient uptake of plasmid DNALipofectamin complexes into the cells. 
Inefficient viral transduction

that can be a result of:

a) Low viral particles being See above for possible generated by the packaging solutions.

cells.

b) Suboptimal concentration

Determine the concentration of polybrene during the of polybrene to be used in transduction process. the cell line of interest with minor toxic effects. For MRC5 fibroblast, $10 \mu \mathrm{g} / \mathrm{ml}$ is the optimal concentration of polybrene for an efficient transduction. Note that some cell lines are particularly sensitive to polybrene, in this case do not use polybrene.

c) Excessive antibiotic used

Determine the antibiotic for selection. sensitivity of the cell line of interest by performing a survival curve. Use the minimum antibiotic required 


\begin{tabular}{|c|c|c|c|}
\hline & & & $\begin{array}{l}\text { to kill non-transduced cells. } \\
\text { For the cell lines described in } \\
\text { this protocol, } 250 \mu \mathrm{M} \text { of } \\
\text { Zeocin }^{\mathrm{TM}} \text { is the optimal } \\
\text { antibiotic concentration. }\end{array}$ \\
\hline \multirow[t]{3}{*}{12} & $\begin{array}{l}\text { Incomplete } \\
\text { mitophagy }\end{array}$ & Loss of CCCP efficiency & $\begin{array}{l}\text { CCCP is photosensitive. It is } \\
\text { critical to aliquot very small } \\
\text { volumes (e.g. } 10 \mu \mathrm{l}) \text { of } \mathrm{CCCP} \text {, } \\
\text { store it at }-20^{\circ} \mathrm{C} \text { and protect it } \\
\text { from light in order to } \\
\text { maintain its efficiency. }\end{array}$ \\
\hline & & $\begin{array}{l}\text { Suboptimal mitochondrial } \\
\text { membrane uncoupling } \\
\text { treatment }\end{array}$ & $\begin{array}{l}\text { Perform a drug concentration } \\
\text { and treatment duration } \\
\text { (including drug refreshment } \\
\text { periodicity) testing, if using a } \\
\text { different cell line to the ones } \\
\text { described in this protocol. }\end{array}$ \\
\hline & & $\begin{array}{l}\text { Suboptimal cell density. Cell } \\
\text { density, prior and during } \\
\text { mitochondria membrane } \\
\text { uncoupling, is a major } \\
\text { determinant of cell viability } \\
\text { upon complete mitophagy. } \\
\text { Low cell confluency, prior } \\
\text { and during uncoupling, may } \\
\text { result in cell death. }\end{array}$ & $\begin{array}{l}\text { Primary fibroblasts require } \\
70-90 \% \text { cell density, prior and } \\
\text { during treatment, for optimal } \\
\text { cell viability post treatment. } \\
\text { However, other cells lines, } \\
\text { particularly immortalized } \\
\text { lines (e.g. HeLa cells) require } \\
\text { lower cell confluency using } \\
\text { this protocol. Determine the } \\
\text { optimal cell density for the } \\
\text { cell line of interest is crucial }\end{array}$ \\
\hline
\end{tabular}


for the success of the

protocol.

Optional: A possibility to avoid cell death is the treatment with caspase inhibitors.

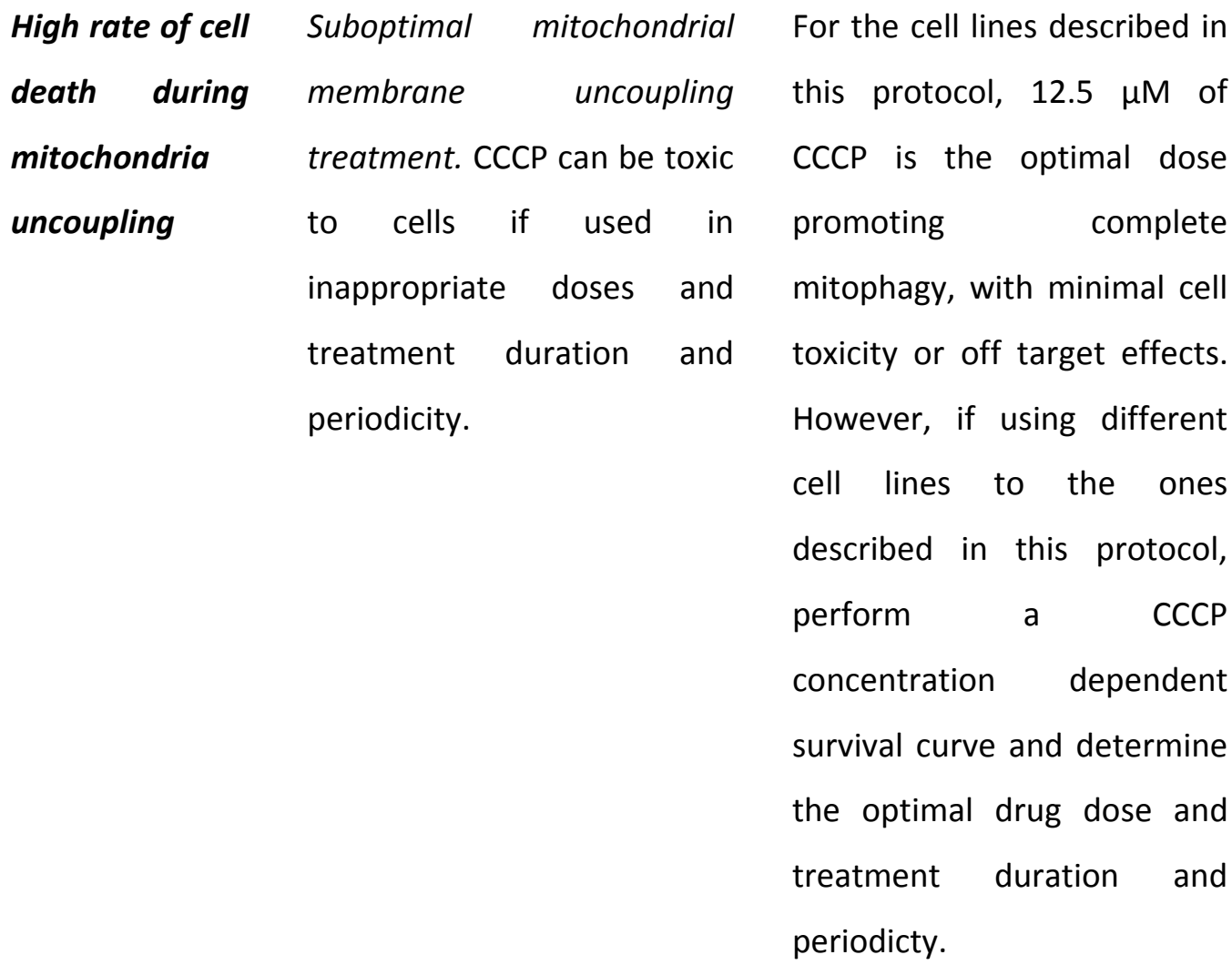

13 High rate of cell Suboptimal cell density. See above (step 12)

death shortly

post complete

mitophagy

17 Premature loss Suboptimal cell density

For primary fibroblasts, cells of cell viability should be $70 \%$ confluent in long-term prior mitochondria 
membrane uncoupling treatment and $90 \%$ confluent after re-seeding cells for experiments post-induction of complete mitophagy.

Suboptimal concentration of For primary fibroblasts, uridine. In long-term supplement the cell culture experiments, it is critical for medium with $50 \mathrm{mg} / \mathrm{ml}$ of cell fitness and viability that uridine. Other cells lines may cells are cultured in medium have different uridine supplemented with uridine requirements, therefore to overcome the limiting determining the uridine pyrimidine levels in concentration for the cell line mitochondria-depleted cells. of interest is fundamental for extension on cell viability in mitochondria-depleted cell. 


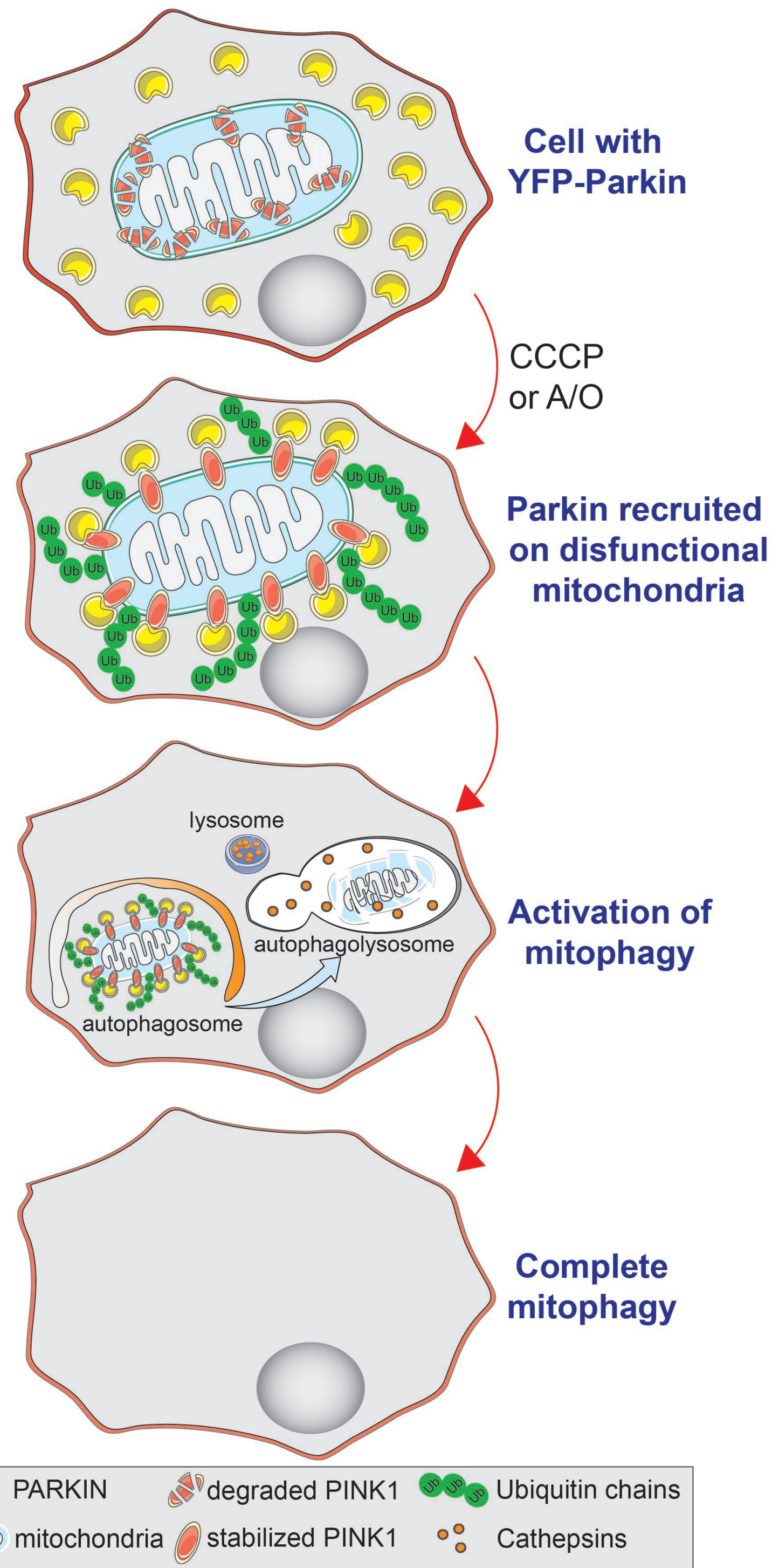



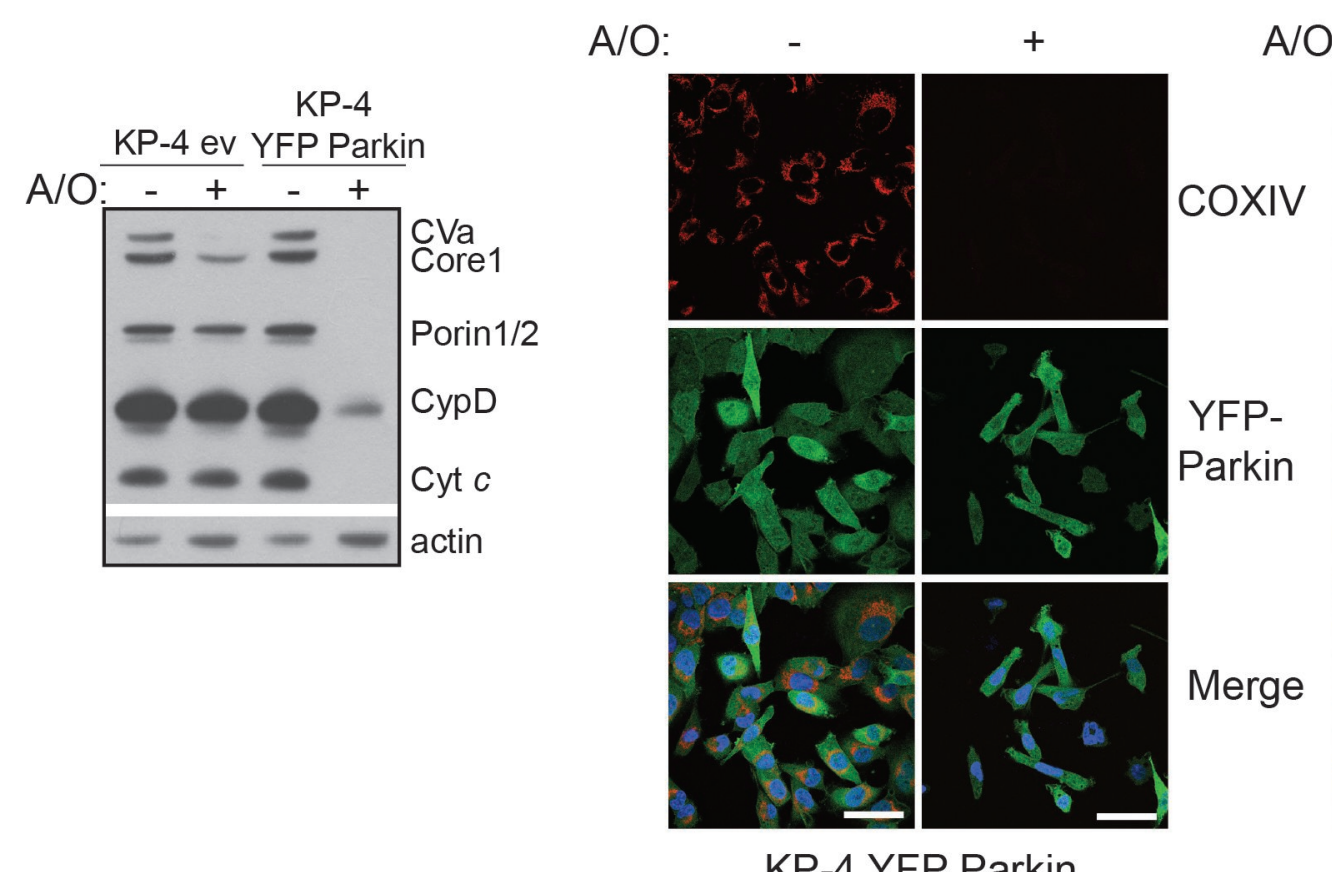

KP-4 YFP Parkin

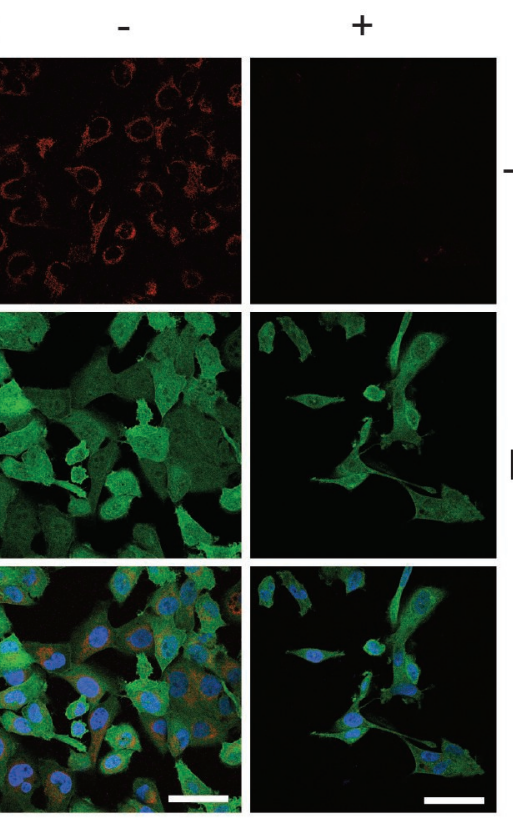

TIMM44

YFP-

Parkin

KP-4 YFP Parkin

D
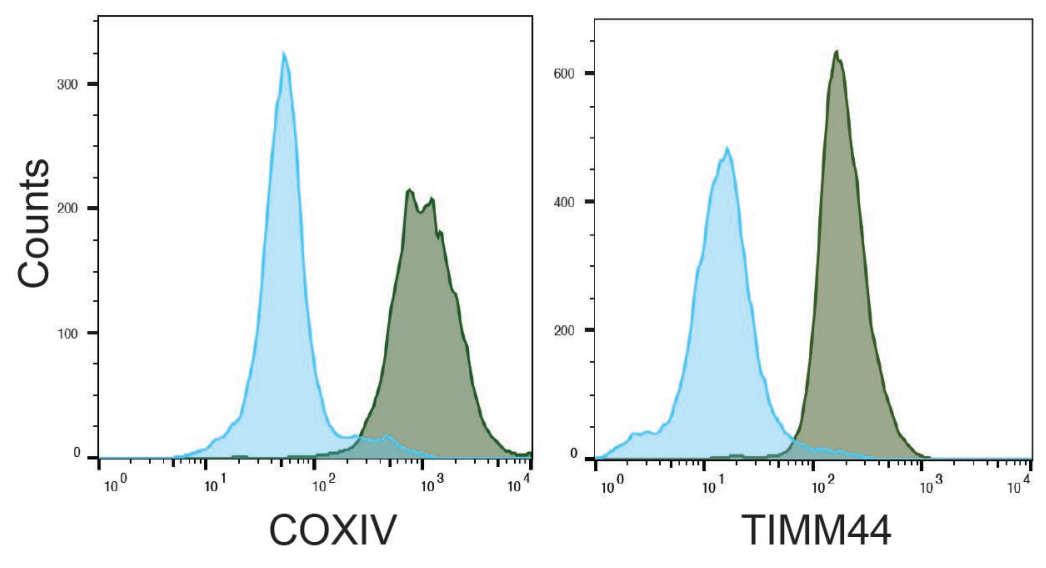

EtOH A/O

$\mathbf{F}$

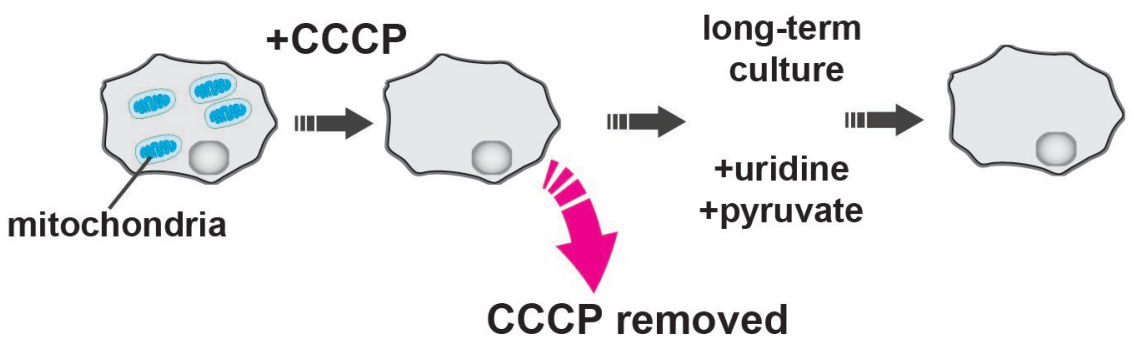

E

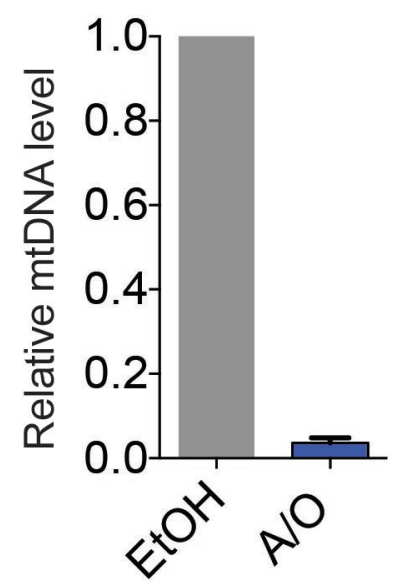

$\mathbf{G}$

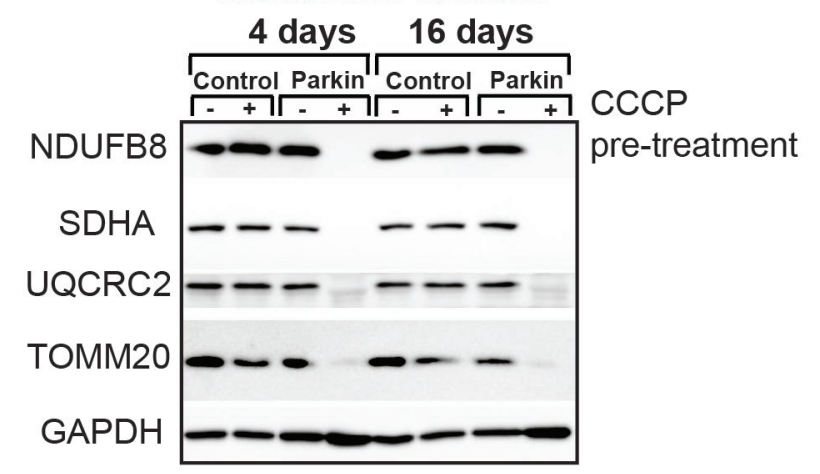

